\title{
Preoperative short course radiotherapy with concurrent and consolidation chemotherapies followed by delayed surgery in locally advanced rectal cancer: preliminary results
}

\author{
Mahdi Aghili, MD', Sarvazad Sotoudeh, MD', Reza Ghalehtaki, MD, \\ Mohammad Babaei, MD¹, Borna Farazmand, MD¹, Mohammad-Sadegh Fazeli, MD², Amir Keshvari, MD², \\ Peiman Haddad, MD', Farshid Farhan, MD \\ 'Radiation Oncology Research Center, Cancer Institute, Tehran University of Medical Sciences, Tehran; \\ ${ }^{2}$ Colorectal Research Center, Imam Khomeini Hospital Complex, Tehran University of Medical Sciences, Tehran, Iran
}

Purpose: This study aimed to assess complications and outcomes of a new approach, that is, combining short course radiotherapy (SRT), concurrent and consolidative chemotherapies, and delayed surgery.

Materials and Methods: In this single arm phase II prospective clinical trial, patients with T3-4 or N+ M0 rectal adenocarcinoma were enrolled. Patients who received induction chemotherapy or previous pelvic radiotherapy were excluded. Study protocol consisted of three-dimensional conformal SRT (25 Gy in 5 fractions in 1 week) with concurrent and consolidation chemotherapies including capecitabine and oxaliplatin. Total mesorectal excision was done at least 8 weeks after the last fraction of radiotherapy. Primary outcome was complete pathologic response and secondary outcomes were treatment related complications. Results: Thirty-three patients completed the planned preoperative chemoradiation and 26 of them underwent surgery (24 low anterior resection and 2 abdominoperineal resection). Acute proctitis grades 2 and 3 were seen in $11(33.3 \%)$ and $7(21.2 \%)$ patients, respectively. There were no grades 3 and 4 subacute hematologic and non-hematologic (genitourinary and peripheral neuropathy) toxicities and perioperative morbidities such as anastomose leakage. Grade 2 or higher late toxicities were observed among 29.6\% of the patients. Complete pathologic response was achieved in 8 (30.8\%) patients who underwent surgery. The 3 -year overall survival and local control rates were $65 \%$ and $94 \%$, respectively.

Conclusion: This study showed that SRT combined with concurrent and consolidation chemotherapies followed by delayed surgery is not only feasible and tolerable without significant toxicity but also, associated with promising complete pathologic response rates.

Keywords: Combined modality therapy, Conformal radiotherapy, Rectal cancer, Iran, Consolidation chemotherapy, Anticancer drug combination, XELOX

\section{Introduction}

Patients with early stage rectal cancer can be treated with radical surgery alone or local resection with or without chemoradiation. However, management of locally advanced rectal cancer is somehow more sophisticated. Surgical

Received 05 April 2017, Revised 15 October 2017, Accepted 30 October 2017

Correspondence: Farshid Farhan, MD, Radiation Oncology Research Center, Cancer Institute, Tehran University of Medical Sciences, Tehran, Iran. Tel: +98-21-61192518, Fax: +98-21-66581633, E-mail: farhan@tums.ac.ir

(c) This is an Open Access article distributed under the terms of the Creative Commons Attribution Non-Commercial License (http://creativecommons.org/ licenses/by-nc/4.0/) which permits unrestricted non-commercial use, distribution, and reproduction in any medium, provided the original work is properly cited.

www.e-roj.org 
management of primary rectal cancer per se is associated with high local and distant recurrence that necessitates a multimodality treatment protocol. Following the study of Sauer et al. [1], it was shown that in German Rectal Cancer Trial, neoadjuvant radiochemotherapy significantly increased local control and overall survival at 10 years and was considered as the standard approach towards locally advanced rectal adenocarcinoma. There are two accepted methods for neoadjuvant radiotherapy: first, mainly North American method also called conventional chemoradiation which includes 45 to 50.4 Gy of radiation in 1.8-2 Gy fractions together with 5 -fluorouracil (5FU) based concurrent chemotherapy. The second method, commonly practiced in Scandinavia, is short course radiotherapy (SRT) that consists of $25 \mathrm{~Gy}$ in 5 consecutive daily fractions followed by immediate surgery within 1 week $[2,3]$. These two approaches do not differ in the rate of local control, overall survival and even perioperative complications as shown in Trans-Tasman Rectal Trial [4]. In addition, this method has been previously proven to be safe and effective in a high burden radiotherapy facility with long patient waiting list [5]. However, some authors believe that a good neoadjuvant treatment should provide tumor downstaging, improved resectability and sphincter preservation in low rectal tumors as well. However, considering the limited interval between radiotherapy and surgery and also lack of concurrent chemotherapy in short course method, less response occurs in neoadjuvant treatment as compared to long course chemoradiation [6].

Due to the fact that SRT is associated with lower cost and duration of treatment, this method cannot be ignored in countries with limited health expenditure like ours. In addition, other investigators also tried to overcome the major drawback of SRT which is its less pathologic complete response (PCR) and sphincter preservation rate as compared to conventional method. In this regard, some authors proposed increasing the interval between radiotherapy and surgery [7] and reported about 10\% increase in pCR in group with delayed surgery. Other investigators tested the addition of preoperative chemotherapy following SRT [8] and delayed surgery with results comparable to conventional chemoradiation. Due to the concerns of increased toxicity, only a few studies are available regarding the addition of concurrent chemotherapy to SRT but with appropriate results and acceptable complications rate $[9,10]$.

Considering the promising results achieved in the earlier mentioned approaches regarding the improvement of SRT flaws, in this study, the authors aimed to assess the ability to induce $\mathrm{pCR}$, feasibility and toxicities by a new approach including neoadjuvant SRT with concurrent and preoperative consolidative chemotherapies followed by delayed surgery.

\section{Materials and Methods}

\section{Study design and participants}

This study is a phase 2 single-arm prospective clinical trial. Patients referred to our radiation oncology ward for neoadjuvant treatment with a pathologic report of rectal adenocarcinoma were recruited in this study. The study design was reviewed and approved by the Research Ethics Committee of Tehran University of Medical Sciences (No. 9111188003 100785), to be in line with declaration of Helsinki. The patients participated voluntarily in this study and all possible complications were disclosed to them. Written inform consent was obtained before any treatment. The trial was registered with Iranian Registry of Clinical Trials (http://www.irct.ir), a regional branch of World Health Organization Clinical Trial Registration (No. IRCT2016121424266N2).

\section{Pretreatment assessment and inclusion/exclusion criteria}

A full clinical and radiologic local staging was performed by physical examination (including digital rectal examination), flexible colonoscopy, pelvic magnetic resonance imaging or rectal endoscopic ultrasonography. Other mandatory diagnostic workup consisted of contrast enhanced thorax and abdominopelvic computed tomography scan, complete blood count, liver and renal function tests and serum carcinoembryonic antigen; positron emission tomography was not in the study protocol because of limited availability. Finally, patients with the following criteria were enrolled for the study: $\mathrm{T} 3, \mathrm{~T} 4$ or lymph node positive $(\mathrm{N}+)$ rectal adenocarcinoma located up to $15 \mathrm{~cm}$ from anal verge, the Eastern Cooperative Oncology Group (ECOG) performance status 0-1. The patients who were candidates of abdominoperineal resection (APR) regardless of response to radiation therapy were also included. Patients with one of the following conditions were excluded from the study: recurrent tumors after previous surgery, synchronous distant metastasis, previous history of pelvic radiotherapy, history of another cancer, impairment of renal function test as much as not to tolerate oxaliplatin or capecitabine, receiving induction chemotherapy and also medical unfitness for surgery. 


\section{Radiotherapy protocol}

All the patients were treated by three-dimensional (3D) conformal radiotherapy with $18 \mathrm{MV}$ photon $\mathrm{X}$-rays. The clinical target volume included the gross tumor and involved nodes together with elective pelvic lymph nodes (presacral, mesorectal and internal iliac lymph nodes in all cases and external and inguinal nodes in appropriate conditions) and entire mesorectum with adequate margins (7-10 $\mathrm{mm}$ for elective and involved nodes, and $2 \mathrm{~cm}$ for primary tumor respecting anatomic boundaries) [11]. The planning target volume (PTV) was defined as clinical target volume plus $1 \mathrm{~cm}$ margin due to patient's positioning and setup error in the center. The PTV received $25 \mathrm{~Gy}$ in 5 daily 5 Gy-fractions for 1 week.

\section{Chemotherapy protocol}

All the patients received concurrent chemotherapy including intravenous oxaliplatin $85 \mathrm{mg} / \mathrm{m}^{2}$ in $\mathrm{d} 1$ and oral capecitabine $825 \mathrm{mg} / \mathrm{m}^{2}$ twice a day in days $1-5$ of radiotherapy. In addition, one cycle of consolidative chemotherapy was prescribed 3-4 weeks after completion of radiotherapy, consisting of intravenous oxaliplatin $130 \mathrm{mg} / \mathrm{m}^{2}$ in day 1 and oral capecitabine $1,000 \mathrm{mg} / \mathrm{m}^{2} / \mathrm{bid}$ in days $1-14$. The adjuvant postoperative chemotherapy was at the discretion of attending physician.

\section{Surgical procedure}

All the participants were referred to surgeon within 6 and 8 weeks after completion of radiotherapy (3-4 weeks after consolidative chemotherapy). The default procedures were low anterior resection (LAR) and APR at the discretion of surgeons. The patients were monitored for perioperative complications by colorectal surgical team for 1 month following operation (in-patient or out-patient) and then referred back with permanent pathology report, to radiation oncology ward.

\section{Complication assessment}

The secondary endpoints were feasibility of combined SRT with concurrent and consolidative chemotherapies. In other words, the complications related to such approach were the center of attention. Thus, patients were evaluated closely for acute (from the beginning of radiotherapy to 1-month post-surgery) and late (after 3 months post-surgery) gastrointestinal, genitourinary and hematologic toxicities using patient reported complaints, physical examination and laboratory studies (complete blood count and renal function tests). The grading of treatment-related toxicities were according to the Common Terminology Criteria for Adverse Events (CTCAE) version 4.0 and highest grade was recorded for each patient. The subjects with proctitis were treated with loperamide, while in cases with bloody discharge, hydrocortisone suppository or 5-aminosalicilates (5ASA) tablets (e.g., mesalamine) were prescribed. They were also evaluated for perioperative complications including anastomosis leakage, delayed surgical wound healing or dehiscence and formation of enterocutaneous, rectovesical or rectovaginal fistula.

\section{Treatment response}

The secondary endpoint was the pathologic response to neoadjuvant treatment. The pathologic response was assessed based on the report done by experienced pathologist in gastrointestinal malignancies. Tumor depth of invasion (ypT) and number of involved lymph nodes (ypN) for tumor and node down-staging were respectively considered. However, the tumor regression grade (TRG) suggested by Dworak et al. [12] was chosen for response evaluation.

\section{Outcomes and analysis}

The primary outcomes were complete pathologic response (ypCR) to neoadjuvant short course radiochemotherapy with delayed surgery and secondary outcomes were feasibility and complications of such treatment. The sample size was calculated based on the formula for detecting 15\% improvement of ypCR from 12.5\% in Stockholm III trial which

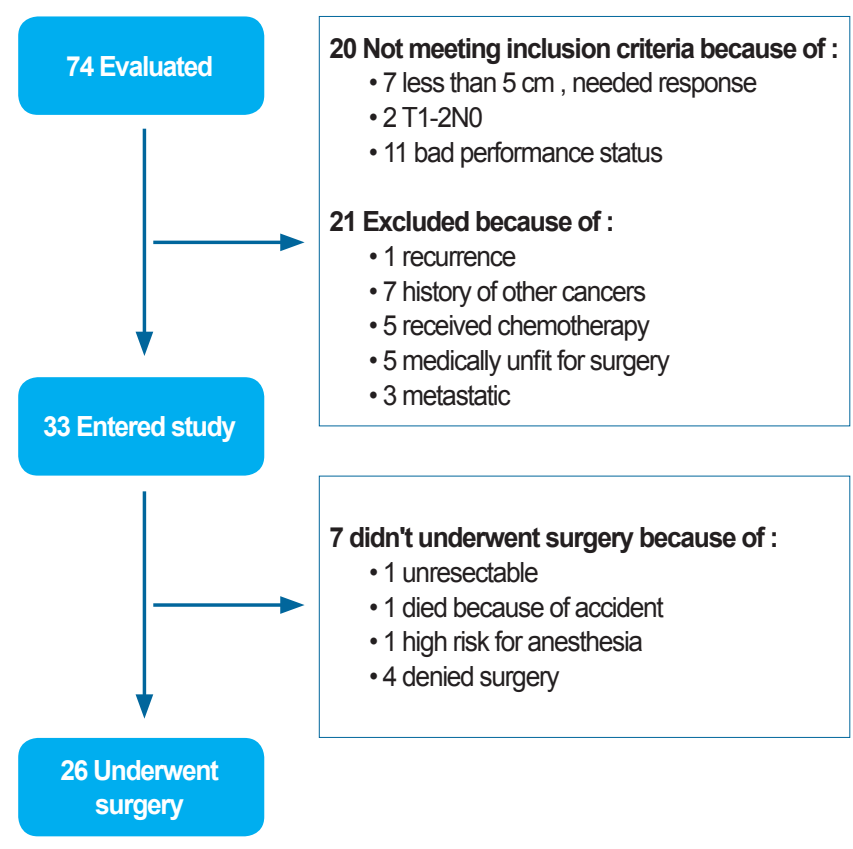

Fig. 1. Allocation diagram. 
Table 1. Patients and tumors characteristics

\begin{tabular}{lc}
\hline \multicolumn{1}{c}{ Characteristic } & Value \\
\hline Age $(y r)$ & $61(32-81)$ \\
Gender & \\
Male & $24(72.7)$ \\
Female & $9(27.3)$ \\
ECOG performance status & \\
0 & $31(93.9)$ \\
1 & $2(6.1)$ \\
Distance from AV (cm) & $6(1-15)$ \\
$<5$ & $5(15.1)$ \\
$5-10$ & $23(69.7)$ \\
$11-15$ & $5(15.1)$ \\
Differentiation & \\
Well (grade 1) & $12(36.3)$ \\
Moderate (grade 2) & $13(39.3)$ \\
Poor (grade 3) & $8(24.2)$ \\
Clinical staging & \\
T3N0 & $8(24.2)$ \\
T3N1 & $17(51.5)$ \\
T3N2 & $5(15.1)$ \\
T4N0 & $1(3)$ \\
T4N1 & $1(3)$ \\
T4N2 & $1(3)$ \\
\hline Values arepresented as medan (range) & \\
\hline &
\end{tabular}

Values are presented as median (range) or number (\%).

Table 2. Frequency of grade 2-3 late treatment related toxicities at 3 months post-surgery

\begin{tabular}{lc}
\hline \multicolumn{1}{c}{ Type of late toxicity events } & Number \\
\hline Erectile dysfunction & 1 \\
Proctitis & 6 \\
Vaginitis & 1 \\
Bowel obstruction & 1 \\
Urinary urge incontinence & 1 \\
Renal failure & 1 \\
\hline
\end{tabular}

was reported in short-course radiotherapy with delayed surgery arm [13]. The power was 80\% and type I error $(\alpha)$ was 0.05. In order to report ypCR, both intentions to treat and per protocol analysis were performed. The stand-point for evaluation of survival rate with Kaplan-Meyer method was the date of the end of radiotherapy.

\section{Results}

\section{Pretreatment characteristics}

From August 2013 to February 2015, a total 33 patients were enrolled in this study (Fig. 1) and their characteristics are shown in Table 1. Mean age ( \pm standard deviation) of the subjects was $58.5 \pm 12.65$ years. The average distance from anal verge was $6.5 \pm 2.79 \mathrm{~cm}$.

\section{Treatment tolerance}

All the subjects completed the course of concurrent chemoradiation successfully. Three patients (12.1\%) did not receive full prescribed dose of consolidative chemotherapy (due to intolerance to oral capecitabine). Fifteen patients (45.5\%) reported no or only grade 1 acute toxicities. Grades 2 and 3 acute proctitis was seen in 11 (33.3\%) and 7 (21.2\%) patients, respectively. No grade 4 acute proctitis was recorded. However, no grade 3 cystitis, hematologic toxicities or peripheral sensory neuropathy were observed.

Twenty-six patients underwent total mesorectal excision (24 LAR and 2 APR). The median interval from end of radiotherapy to surgery was 10 months. Amongst others who failed to undergo surgery, 1 patient died in a car crash before surgery, 4 refused the operation despite primary consent, 1 had an unresectable tumor in pre-surgical evaluation (which was unresectable before radiotherapy as well), and the last 1 was medically unfit for surgery due to cardiac disease that progressed during chemoradiation. During 1-month postoperative period, only an event of grade 3 surgical site infection occurred in one diabetic patient. Eleven events of late treatment related to grades 2 or 3 toxicity occurred in 8 patients (24.2\%) (Table 2).

\section{Pathologic response}

Complete pathologic response (TRGO) was reported in 8 patients (24.2\%), partial response (TRG1 and TRG2) was reported in 6 patients (18.2\%) and poor response (TRG3) was reported in 12 patients (36.4\%) out of the 33 study subjects. Among the subjects who underwent surgery, the rates of complete, partial, and poor pathologic response were $30.8 \%$ $23.0 \%$, and $46.2 \%$, respectively. All the patients enjoyed R0 resection.

\section{Treatment outcomes}

The 3-year overall survival (OS) and disease-free survival (DFS) in all the subjects was 60\% and 52\%, respectively (Figs. 2 and $3)$. The local control (LC) and distant control (DC) rates were $84 \%$ and 68\%, respectively, among all the study subjects. However, among the subjects who underwent surgery per protocol, the rates of OS, DFS, LC, and DC were 65\%, 55\%, $94 \%$, and $63 \%$, respectively. 


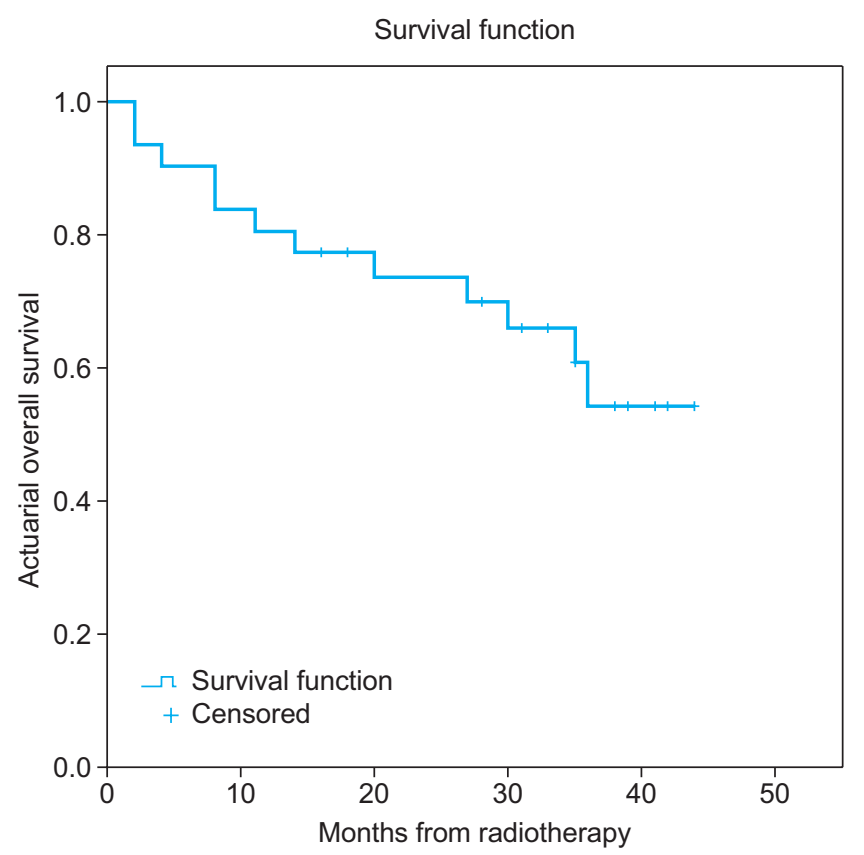

Fig. 2. Actuarial overall survival rate.

\section{Discussion and Conclusion}

Neoadjuvant treatment is the standard of care for patients with locally advanced rectal cancer. Chemoradiation or SRT are both widely accepted as neoadjuvant treatments and can be practiced as standard protocols for improving $L C$ in this group of patients. To address the differences in these approaches, two phase III randomized trials were conducted and the results are available. Polish trial and Trans-Tasman Radiation Oncology (TROG) group did not show a significant difference in survival and local recurrence rate between chemoradiation and SRT $[4,14]$.

TROG trial showed that SRT had significantly less acute adverse events as compared to long course chemoradiotherapy, while the post-operative complications were comparable [15]. Nevertheless, the major concern that keep colorectal oncologist from concurrent short course chemoradiotherapy is the fear of escalated treatment-related acute and late complications [6]. There is agreement among various studies that by lengthening the interval between radiotherapy and surgery, these increased risk of complications is negligible as compared to long course chemoradiotherapy [16]. The concept of delayed surgery after SRT was also tested in Stockholm III trial and it was shown that the risk of post-operative complications are significantly lower in SRT with delayed surgery group as compared to immediate surgery (38\% vs. $50 \%)$ but the oncological outcomes were

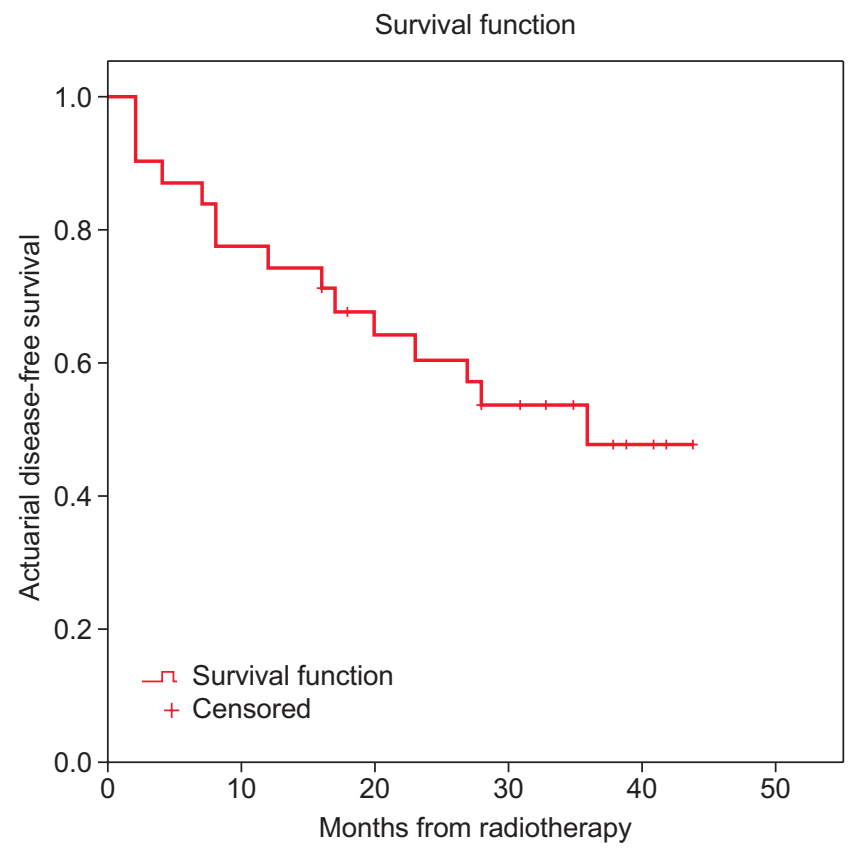

Fig. 3. Actuarial disease-free survival rate.

statistically equal [17].

Some studies aimed to test chemotherapy in a neoor adjuvant sequence to radiotherapy before surgery. For instance, in Dutch rectal cancer trial, rectal cancer patients with synchronous metastasis to liver showed acceptable complication rate with preoperative chemotherapy following SRT [18]. Accordingly, Japanese investigators showed the safety of induction chemotherapy plus cetuximab and SRT [19]. However, there are limited experiences with concurrent chemotherapy and SRT. In one of these rare instances, KROG 10-01 phase II trial, the rate of grade 3 or more toxicities were considerably high (about 38\%) [20]. In contrast, another Korean study by Chung et al. [9] showed similar toxicities between short and long course chemoradiotherapies. This difference regarding toxicities could be due to the selection of concurrent chemotherapy regimen. The KROG 10-01 trial utilized bolus 5FU, while the latter utilized infusional 5FU which is expectedly less toxic. Interestingly, in their subsequent trial on the so-called KROG 11-02, same authors changed the regimen to oral capecitabine and this time, reported more acceptable safety profiles [21]. In our study, concurrent oral capecitabine in adjunct to intravenous oxaliplatin regimen was used to improve compliance and safety together with high pathologic response rate, and both goals were met fortunately. Another explanation for the acceptable rates of acute and late complications could be the differences among ethnicities 
regarding response to chemotherapy $[22,23]$. Perhaps, the Caucasian unlike Asian populations are more resistant to concurrent 5FU based chemotherapy regimens.

Apart from the issue of feasibility and tolerance, a relatively high rate of about $31 \%$ of complete pathologic response (cPR) was observed among the patients who underwent extirpative surgery. Three simultaneous deviations from routine SRT were attempted which could explain the promising results in terms of cPR including delayed surgery, concurrent and consolidative chemotherapies. In orthodox SRT method that is practiced first in Sweden, patients would undergo total mesorectal excision within 1 week after completion of radiotherapy sessions. With early surgery, evaluating the ultimate pathologic response is not too possible, the optimal response may be difficult to achieve and the rate of positive margin will be expectedly high. However, if one can postpone the surgery for some 4-6 weeks, the pathologic response would be theoretically more mature even in primarily unresectable appearing cases [24]. Response to radiotherapy is a continuous process and the optimum interval between completion of radiotherapy and surgery is not clear [25]. Beppu et al. [16] showed that SRT with delayed surgery is non-inferior to long course chemoradiation [26] in terms of down-staging effects and complications. A metaanalytical study also proved that by delaying surgery, the rate of cPR would be $10 \%$ greater than that of earlier surgery.

Besides the role of delayed surgery, the addition of concurrent chemotherapy to conventional fractionated radiotherapy and consolidative chemotherapy in the resting period between radiation completion and surgery, both have significantly increased the $P C R$ rate and $L C$ in locally advanced rectal cancer and have acceptable and tolerable toxicity $[27,28]$. Following these successful observations, some authors implemented chemotherapy as adjunct to neoadjuvant SRT. Myerson et al. [8] tested the efficacy of consolidation chemotherapy in the interval between SRT and surgery. The rate of pCR and 2-year LC were 25\% and 95\%, respectively. Some investigators assume that, like gastric cancer, neoadjuvant chemotherapy might be associated with improved survival. Currently, this hypothesis is being tested in adjunct to SRT in RAPIDO trial [29]. However, the above studies used chemotherapy in a sequential fashion with SRT but in trials implementing concurrent chemotherapy protocols, the rate of cPR ranged from $1.4 \%$ to $21.1 \%[9,10,20,21]$. It is noteworthy that regimens that contain oral capecitabine are associated with absolutely better and higher responses than bolus and infusional 5FU. However, our superior results could be explained by the addition of oxaliplatin to oral capecitabine, that has been shown to significantly improve response rate (TRGO and TRG1) both in ACCORD trial [30] and in our center previous experience [31]. In order to introduce a new concurrent regimen with SRT, the results of several metaanalysis were used, suggesting the benefit of adding oxaliplatin to $5 \mathrm{FU}$ based concurrent long course chemoradiotherapy [3234].

The main limitation of this study is the small size and nonrandomized design. Another limitation that is worth mentioning is the need for longer follow-up for late effects of radiation and the rate of $L C$. The observed promising results need to be tested in a randomized manner in comparison with conventional long course chemoradiotherapy.

In conclusion, this study showed that SRT with concurrent chemotherapy and consolidation chemotherapy with delayed surgery are not only feasible and tolerable without significant toxicity but are also associated with promising pathologic response rates. However, this is a small and nonrandomized one-arm study with relatively short follow-up which needs longer assessments for monitoring of late complications. Further investigation is needed to compare this protocol with conventional fractionated protocol in a phase III randomized trial.

\section{Conflict of Interest}

No potential conflict of interest relevant to this article was reported.

\section{References}

1. Sauer $R$, Becker $H_{1}$ Hohenberger W, et al. Preoperative versus postoperative chemoradiotherapy for rectal cancer. N Engl J Med 2004;351:1731-40.

2. Cedermark $B$, Johansson $H$, Rutqvist LE, Wilking N. The Stockholm I trial of preoperative short term radiotherapy in operable rectal carcinoma: a prospective randomized trial. Stockholm Colorectal Cancer Study Group. Cancer 1995;75:2269-75.

3. Martling A, Holm T, Johansson H, Rutqvist LE, Cedermark B; Stockholm Colorectal Cancer Study Group. The Stockholm II trial on preoperative radiotherapy in rectal carcinoma: long-term follow-up of a population-based study. Cancer 2001;92:896-902.

4. Ngan SY, Burmeister B, Fisher RJ, et al. Randomized trial of short-course radiotherapy versus long-course chemoradiation comparing rates of local recurrence in patients with $\mathrm{T} 3$ rectal 
cancer: Trans-Tasman Radiation Oncology Group trial 01.04. J Clin Oncol 2012;30:3827-33.

5. Farhan F, Fazeli MS, Samiei F, et al. Morbidity and mortality following short course preoperative radiotherapy in rectal carcinoma. Acta Med Iran 2015;53:627-32.

6. Minsky BD. Short-course radiation versus long-course chemoradiation for rectal cancer: making progress. J Clin Oncol 2012;30:3777-8.

7. Bujko K, Partycki M, Pietrzak L. Neoadjuvant radiotherapy (5 $\times 5$ Gy): immediate versus delayed surgery. Recent Results Cancer Res 2014;203:171-87.

8. Myerson RJ, Parikh PJ, Tan B, et al. A single-institution phase II trial of five fractions of radiotherapy followed by four courses of FOLFOX chemotherapy as preoperative therapy for rectal adenocarcinoma. J Clin Oncol 2012;30(4_Suppl):553.

9. Chung MJ, Kim DW, Chung WK, et al. Preoperative short- vs. long-course chemoradiotherapy with delayed surgery for locally advanced rectal cancer. Oncotarget 2016;8:60479-86.

10. Rasulov AO, Gordeyev SS, Barsukov YA, et al. Short-course preoperative radiotherapy combined with chemotherapy, delayed surgery and local hyperthermia for rectal cancer: a phase II study. Int J Hyperthermia 2017:1-9.

11. Myerson RJ, Garofalo MC, El Naqa I, et al. Elective clinical target volumes for conformal therapy in anorectal cancer: a radiation therapy oncology group consensus panel contouring atlas. Int J Radiat Oncol Biol Phys 2009;74:824-30.

12. Dworak O, Keilholz L, Hoffmann A. Pathological features of rectal cancer after preoperative radiochemotherapy. Int J Colorectal Dis 1997;12:19-23.

13. Pettersson $D$, Cedermark $B$, Holm $T$, et al. Interim analysis of the Stockholm III trial of preoperative radiotherapy regimens for rectal cancer. Br J Surg 2010;97:580-7.

14. Bujko K, Nowacki MP, Nasierowska-Guttmejer A, Michalski W, Bebenek M, Kryj M. Long-term results of a randomized trial comparing preoperative short-course radiotherapy with preoperative conventionally fractionated chemoradiation for rectal cancer. Br J Surg 2006;93:1215-23.

15. Ansari N, Solomon MJ, Fisher RJ, et al. Acute adverse events and postoperative complications in a randomized trial of preoperative short-course radiotherapy versus long-course chemoradiotherapy for $\mathrm{T3}$ adenocarcinoma of the rectum: Trans-Tasman Radiation Oncology Group Trial (TROG 01.04). Ann Surg 2017;265:882-8.

16. Beppu N, Matsubara N, Kakuno A, et al. Feasibility of modified short-course radiotherapy combined with a chemoradiosensitizer for T3 rectal cancer. Dis Colon Rectum 2015;58:479-87.
17. Erlandsson J, Holm T, Pettersson D, et al. Optimal fractionation of preoperative radiotherapy and timing to surgery for rectal cancer (Stockholm III): a multicentre, randomised, non-blinded, phase 3, non-inferiority trial. Lancet Oncol 2017;18:336-46.

18. van Dijk TH, Tamas K, Beukema JC, et al. Evaluation of shortcourse radiotherapy followed by neoadjuvant bevacizumab, capecitabine, and oxaliplatin and subsequent radical surgical treatment in primary stage IV rectal cancer. Ann Oncol 2013;24:1762-9.

19. Beppu N, Yoshie H, Kimura F, et al. The short-term outcomes of induction SOX (S-1 + oxaliplatin) \pm cetuximab chemotherapy followed by short-course chemoradiotherapy in patients with poor-risk locally advanced rectal cancer. Surg Today 2016;46:1123-31.

20. Yeo SG, Oh JH, Kim DY, et al. Preoperative short-course concurrent chemoradiation therapy followed by delayed surgery for locally advanced rectal cancer: a phase 2 multicenter study (KROG 10-01). Int J Radiat Oncol Biol Phys 2013;86:34-9.

21. Lee JH, Kim JG, Oh ST, et al. Two-week course of preoperative chemoradiotherapy followed by delayed surgery for rectal cancer: a phase II multi-institutional clinical trial (KROG 1102). Radiother Oncol 2014;110:150-4.

22. O'Donnell PH, Dolan ME. Cancer pharmacoethnicity: ethnic differences in susceptibility to the effects of chemotherapy. Clin Cancer Res 2009;15:4806-14.

23. Phan VH, Moore MM, McLachlan AJ, Piquette-Miller $M, X U$ $\mathrm{H}, \mathrm{Clarke} \mathrm{SJ}$. Ethnic differences in drug metabolism and toxicity from chemotherapy. Expert Opin Drug Metab Toxicol 2009;5:243-57.

24. Hatfield $P$, Hingorani $M$, Radhakrishna $G$, et al. Short-course radiotherapy, with elective delay prior to surgery, in patients with unresectable rectal cancer who have poor performance status or significant co-morbidity. Radiother Oncol 2009;92:210-4.

25. Kwak YK, Kim K, Lee JH, et al. Timely tumor response analysis after preoperative chemoradiotherapy and curative surgery in locally advanced rectal cancer: a multi-institutional study for optimal surgical timing in rectal cancer. Radiother Oncol 2016;119:512-8.

26. Beppu N, Matsubara N, Noda M, et al. Short-course radiotherapy with delayed surgery versus conventional chemoradiotherapy: a comparison of the short- and longterm outcomes in patients with T3 rectal cancer. Surgery 2015;158:225-35.

27. Habr-Gama A, Perez RO, Sabbaga J, Nadalin W, Sao Juliao GP, 
Gama-Rodrigues J. Increasing the rates of complete response to neoadjuvant chemoradiotherapy for distal rectal cancer: results of a prospective study using additional chemotherapy during the resting period. Dis Colon Rectum 2009;52:1927-34.

28. Gerard JP, Conroy T, Bonnetain F, et al. Preoperative radiotherapy with or without concurrent fluorouracil and leucovorin in T3-4 rectal cancers: results of FFCD 9203. J Clin Oncol 2006;24:4620-5.

29. Nilsson PJ, van Etten B, Hospers GA, et al. Short-course radiotherapy followed by neo-adjuvant chemotherapy in locally advanced rectal cancer: the RAPIDO trial. BMC Cancer 2013;13:279.

30. Gerard JP, Azria D, Gourgou-Bourgade S, et al. Comparison of two neoadjuvant chemoradiotherapy regimens for locally advanced rectal cancer: results of the phase III trial ACCORD 12/0405-Prodige 2. J Clin Oncol 2010;28:1638-44.
31. Haddad $P_{1}$ Miraie M, Farhan $F_{1}$ et al. Addition of oxaliplatin to neoadjuvant radiochemotherapy in MRI-defined T3, T4 or $\mathrm{N}_{+}$ rectal cancer: a randomized clinical trial. Asia Pac J Clin Oncol 2017;13:416-22.

32. van der Pols JC, Russell A, Bauer U, Neale RE, Kimlin MG, Green AC. Vitamin D status and skin cancer risk independent of time outdoors: 11-year prospective study in an Australian community. J Invest Dermatol 2013;133:637-41.

33. Yang YJ, Cao L, Li ZW, et al. Fluorouracil-based neoadjuvant chemoradiotherapy with or without oxaliplatin for treatment of locally advanced rectal cancer: an updated systematic review and meta-analysis. Oncotarget 2016;7:45513-24.

34. Zheng J, Feng $X, H u$ W, Wang J, Li Y. Systematic review and meta-analysis of preoperative chemoradiotherapy with or without oxaliplatin in locally advanced rectal cancer. Medicine (Baltimore) 2017;96:e6487. 